\title{
Factores asociados a la sobrevida en pacientes con tumor de Wilms
}

\author{
Factors associated with survival in patient with Wilms tumor
}

\author{
Natalia Herrera-Toro ${ }^{1} \mathbb{D}$, Laura Peña-Aguirre ${ }^{2} \mathbb{D}$, Federico Molina ${ }^{3}$ (D)
}

Médica, especialista en Cirugía pediátrica, magister en Urología pediátrica, Hospital Pablo Tobón Uribe; profesora, Cirugía y
urología pediátrica, Universidad de Antioquia, Medellín, Colombia.
2 Médica general, Sura EPS, La Ceja, Colombia.
3 Médico, especialista en Toxicología, doctor en Epidemiología; profesor, Instituto Tecnológico de Antioquia, Medellín, Colombia.

\section{Resumen}

Introducción. El tumor de Wilms es el segundo tumor abdominal más frecuente en la edad pediátrica y responde por más del $90 \%$ de los tumores renales en pediatría. A pesar de que la sobrevida descrita es mayor del $90 \%$, en nuestro medio encontramos que solo alcanza al $70 \%$, por lo que deseamos evaluar cuáles son los factores asociados con dichos resultados desfavorables, con el fin de implementar medidas para mejorar la sobrevida de nuestros pacientes.

Métodos. Se realizó un estudio observacional, transversal, en dos centros de alto nivel de atención, que incluyó una muestra de 84 pacientes menores de I5 años, con diagnóstico de tumor de Wilms.

Resultados. Los factores que se asociaron significativamente con un aumento en la probabilidad de morir fueron: no completar el protocolo de quimioterapia, (OR 34; IC $95 \%$ 3,7-3I2; $p$ 0,000) y presentar recidiva tumoral (OR 35,7; $\mathrm{IC}_{95} \%$ 6,9-I84; $p$ o,ooo). Otros factores que aumentaron esta probabilidad sin alcanzar a ser significativos, pero mostrando una evidente tendencia fueron: presentación bilateral ( $\mathrm{OR} 4, \mathrm{I} ; \mathrm{IC}_{95} \%$ 0,6-5,5; $p$ o, I47), complicaciones quirúrgicas (OR 3,2; $\mathrm{IC}_{95 \%}$ O,7-I4,6; $p$ o,I36), compromiso de ganglios linfáticos en tomografía $\left(\mathrm{OR}_{2,4}\right.$; IC $95 \%$ O,78,$4 ; p$ 0,139) y las metástasis a distancia (OR 2,5; $\mathrm{IC}_{95} \% 0,7-9 ; p$ 0,I43).

Discusión. La sobrevida de nuestros niños con tumor de Wilms es menor que la reportada en la literatura mundial, siendo la falla en terminar la quimioterapia, la recidiva y la necesidad de cirugía bilateral, los factores asociados con este desenlace.

Palabras clave: tumor de Wilms; nefroblastoma; cirugía; urología; oncología quirúrgica; supervivientes de cáncer.

Fecha de recibido: 03/02/2020 - Fecha de aceptación: 24/05/2020

Correspondencia: Natalia Herrera-Toro, Calle 33 C \# 88A - 115. Teléfono: 5593922.

Correo electrónico: nataherrerat@gmail.com

Citar como: Herrera-Toro N, Peña-Aguirre L, Molina F. Factores asociados a la sobrevida en pacientes con tumor de Wilms. Rev Colomb Cir. 2020;35:621-9. https://doi.org/10.30944/20117582.556

Este es un artículo de acceso abierto bajo una Licencia Creative Commons - BY-NC-ND https://creativecommons.org/licenses/by-ncnd/4.0/deed.es 


\begin{abstract}
Introduction. Wilms tumor is the second most frequent abdominal tumor in pediatric age, and it accounts for more than $90 \%$ of kidney tumors in pediatrics. Although the described survival is greater than $90 \%$, in our setting we find that it only reaches $70 \%$. Our objective was to evaluate the factors associated with these unfavorable results, in order to implement measures to improve the survival of our patients.
\end{abstract}

Methods. An observational, cross-sectional study was conducted in two tertiary medical centers, which included a sample of 84 patients under 15 years of age with a diagnosis of Wilms tumor.

Results. The factors that were significantly associated with an increase in the probability of dying were not completing the chemotherapy protocol (OR 34; ${ }_{95 \%} \mathrm{CI} 3.7-312 ; \mathrm{p}$ 0.000) and presenting tumor recurrence (OR $35.7 ;{ }_{95 \%} \mathrm{CI}$ 6.9-I84; $\mathrm{p}$ 0.000). Other factors that increased this probability without being significant, but showing an evident trend were: bilateral presentation (OR 4.I; ${ }_{95 \%} \mathrm{CI}$ 0.6-5.5; p o.I47), surgical complications (OR 3.2; ${ }_{95 \%} \mathrm{CI}$ 0.7-I4.6; $\mathrm{p}$ o.I36), lymph node involvement in tomography (OR 2.4; $95 \% \mathrm{CI} 0.7-8.4 ; \mathrm{p}$ 0.I39) and distant metastases (OR $2.5 ;{ }_{95 \%} \mathrm{CI}$ 0.7-9; p 0.I43).

Discussion. The survival of the children with Wilms tumor in our study was lower than that reported in the world literature, with failure to complete chemotherapy, recurrence and the need for bilateral surgery being the factors associated with this outcome.

Keywords: Wilms tumor; nephroblastoma; surgery; urology; surgical oncology; cancer survivors.

\section{Introducción}

El tumor de Wilms es el segundo tumor abdominal más frecuente en la edad pediátrica y ocasiona más del $90 \%$ de los tumores renales en pediatría ${ }^{\text {. }}$ En Estados Unidos, el tumor de Wilms tiene una incidencia de 8,I casos por millón por año ${ }^{2,3}$ y en Colombia, los registros del Instituto Nacional de Cancerología reportan una frecuencia de 9,3\% para nefroblastoma, con 3,2 casos por millón de habitantes por año ${ }^{4}$.

La sobrevida en pacientes con tumor de Wilms ha mejorado en las últimas décadas en países desarrollados, gracias a los grupos colaborativos que han enfocado su estudio al empleo de quimioterapia multimodal, al desarrollo de técnicas quirúrgicas y al reconocimiento del papel de la radioterapia, aumentando de $73 \%$ en 1975 , a $93 \%$ en el 20I2, hasta llegar al $95 \%$ en la actualidad 5 . Sin embargo, en nuestro medio, los resultados son menos satisfactorios. En un estudio se encontró que Io8 meses después del diagnóstico, la supervivencia es del 7I \% y que el tiempo promedio de supervivencia libre de recaída es de 97 meses 5 .

El objetivo de este estudio fue evaluar cuáles son los factores asociados con los resultados de supervivencia desfavorables, con el fin de implementar medidas para mejorar la sobrevida de nuestros pacientes con tumor de Wilms.

\section{Métodos}

Se realizó un estudio observacional, transversal, que incluyó una muestra de 84 pacientes menores de I5 años, con diagnóstico de tumor de Wilms, tratados en el servicio de hematooncología infantil de dos hospitales de alto nivel de atención, en Medellín, Colombia, entre el primero de enero del 2005 y el 3I de mayo del 2018.

Para definir los factores asociados con la sobrevida a dos años, se evaluaron variables sociodemográficas (lugar de residencia y sexo), clínicas (presentación clínica, lateralidad, antecedente de malformación), patológicas (patología tumoral, biopsia previa, presencia de necrosis e histología), quirúrgicas (resecabilidad, procedimiento realizado, bilateralidad, contención tumoral y complicaciones), quimioterapia (protocolo utilizado, terminación completa del protocolo), radioterapia, recidiva y hallazgos tomográficos.

Para la recolección de la información se revisaron las historias clínicas de las especialidades 
de cirugía pediátrica, oncología y nefrología infantil durante el periodo determinado.

Para el análisis estadístico se construyó una base de datos en Excel, con las reglas de validación pertinentes para garantizar la calidad de los datos, y se hicieron los cálculos utilizando el programa SPSS versión 22. Las variables cualitativas se presentan con frecuencias absolutas y relativas y las cuantitativas con medidas de tendencia central. Los porcentajes se calcularon sobre el número de pacientes que tenían datos en cada variable.

Se utilizó estadística bivariada aplicando tablas de contingencia con prueba de independencia $\mathrm{chi}^{2}$ al $5 \%$, para medir la relación de las variables con la sobrevida a dos años. Se obtuvieron los odds ratio (OR) ajustados mediante un análisis de regresión logística. Se elaboraron varios modelos con las variables de importancia epidemiológica, en los que también se observó el aporte de las variables independientes que fueron relevantes desde el punto de vista estadístico, con un valor de $p$ inferior a 0,25 . Se excluyeron del análisis las variables independientes cuya correlación sugirió colinealidad y se hicieron pruebas para evaluar el ajuste a través de la razón de verosimilitud.

\section{Resultados}

Se evaluaron en total 84 niños con tumor de Wilms en los hospitales Pablo Tobón Uribe y San Vicente Fundación, entre el primero de enero del 2005 y el 3I de mayo del 20I8. Solo se pudo realizar el seguimiento para establecer la sobrevida a los dos años del diagnóstico en 6I de ellos, la cual fue del $70,5 \%(n=43)$.

Se encontró que 48 pacientes $(57,8 \%$ ) provenía de Medellín y 43 (5I,2 \%) eran hombres. En 6I casos (73,5\%) el motivo de consulta fue el síntoma de masa abdominal, acompañada de otros síntomas. El compromiso fue unilateral y no hubo antecedentes de malformaciones congénitas en 78 pacientes (92,9\%) cada uno. Adicionalmente, en la patología se identificó predominio blastemal en 23 casos (53,5\%), necrosis en once ( $17,7 \%)$ e histología desfavorable en siete $(8,8 \%$ ) (tabla I).
En cuanto a los aspectos quirúrgicos (tabla 2), se encontró que en 5I $(60,7 \%)$ pacientes el tumor era resecable. En 78 casos $(96,3 \%)$ se hizo nefrectomía unilateral, y en 5I $(65,4 \%)$ de estos, el abordaje fue nefrectomía unilateral prequimioterapia. Solo tres $(3,7 \%)$ pacientes requirieron abordaje bilateral y diez (I2,3\%) presentaron complicaciones durante la cirugía.

En la tomografía computarizada se encontró que $23(31, \mathrm{I} \%)$ presentaron compromiso ganglionar, $\mathrm{I} 7(\mathbf{2 2 , \mathrm { I }} \%)$ tenía metástasis a distancia y 8 (Io,4 \%) trombo mural. En relación con la quimioterapia, el protocolo más utilizado fue el del National Wilms' Tumor Study (NWTS) en 58 pacientes (70,7\%). Lograron completar el protocolo 57 pacientes $(83,8 \%)$ y $20(27,4 \%)$ presentaron recidiva tumoral.

En la tabla 3 se presentan los factores que se asociaron significativamente con un aumento en la probabilidad de morir: no completar el protocolo de quimioterapia (OR 34; $\mathrm{IC}_{95} \% 3,7-3 \mathrm{I2}$; $p$ o,00o) y presentar recidiva tumoral (OR 35,7; $\mathrm{IC}_{95 \%} 6,9-\mathrm{I} 84 ; p$ o,ooo). Otros factores que aumentaron esta probabilidad, sin alcanzar a ser estadísticamente significativos, pero mostrando una tendencia en este sentido, fueron: tumor bilateral (OR 4,I; IC $\mathrm{I}_{95} \mathrm{0}, 6-5,5 ; p$ o,I47), complicaciones quirúrgicas (OR 3,2; $\mathrm{IC}_{95 \%}$ 0,7-I4,6; $p$ o,I36), compromiso de ganglios linfáticos en la tomografía (OR 2,$4 ; \mathrm{IC}_{95 \%} 0,7-8,4 ; p$ 0,I39) y metástasis a distancia (OR 2,5; $\mathrm{IC}_{95 \%}$ 0,7-9; $p$ o,I43). Las demás características evaluadas no se asociaron significativamente con la probabilidad de morir.

El modelo final de regresión logística estuvo compuesto por las siguientes variables: no completar el protocolo de quimioterapia (OR II,2; IC $_{95 \%}$ 0,4-329,9; $p$ o,I6I), recidiva tumoral (OR 7I,5; IC $95 \% 6,05-845 ; p$ o,oII) y necesidad de cirugía bilateral (OR 28; IC $95 \%$ I,2-I244; $p$ 0,O4I). Se calculó que el 7I \% de la variabilidad en la sobrevida se explicó por estos tres factores $\left(\mathrm{R}^{2}\right.$ de Nagelkerke 0,7I).

\section{Discusión}

Los factores pronósticos para el tumor de Wilms según el grupo colaborativo del Children's Onco- 
logy Group (COG) son estadio tumoral, histología, edad, peso del tumor, respuesta de los nódulos pulmonares y pérdida de heterocigocidad (LOH) de Ip y I6q, mientas que los de la Sociedad Internacional de Oncología Pediátrica (SIOP) incluyen edad, histología, volumen del tumor y respuesta a la terapia. La respuesta a la quimioterapia se ha definido como la disminución del tamaño tumoral, el cambio en la histología $\mathrm{y}$, en los casos de estadio IV, la desaparición de las metástasis.

Con respecto a la edad, los niños menores de 2 años tienen mejores resultados comparados con los de 2 a 4 años ( $95 \%$ de sobrevida libre de evento a 5 años vs. $88 \%$ ). En otros estudios se reporta una sobrevida del $96 \%$ en menores de I año y $80 \%$ en niños entre I y 2 años ${ }^{6}$. Sin embargo, en el trabajo africano de Aronson y Hadley se describió que sólo en estadio I con histología favorable los menores de 4 años tienen mejores resultados que los mayores ${ }^{7}$.

El volumen tumoral puede considerarse un factor pronóstico en tumores de riesgo intermedio, excluyendo los de predominio epitelial o estromal $^{8}$. Teniendo en cuenta el tamaño tumoral, para el punto de corte de $500 \mathrm{ml}$ en pacientes

Tabla 1. Características de los pacientes con tumor de Wilms de la población en estudio.

\begin{tabular}{|c|c|c|c|}
\hline Variable & Categoría & $\mathbf{n}$ & $\%$ \\
\hline \multirow[t]{4}{*}{ Lugar de residencia } & Medellín y área metropolitana & 48 & 57,8 \\
\hline & Antioquia & 25 & 30,1 \\
\hline & Otros departamentos & 10 & 12,0 \\
\hline & Sin dato & 1 & \\
\hline \multirow[t]{2}{*}{ Sexo } & Masculino & 43 & 51,2 \\
\hline & Femenino & 41 & 48,8 \\
\hline \multirow[t]{2}{*}{ Presentación Clínica } & $\begin{array}{l}\text { Masa abdominal y otra } \\
\text { sintomatología }\end{array}$ & 61 & 73,5 \\
\hline & $\begin{array}{l}\text { Dolor abdominal u otra } \\
\text { sintomatología }\end{array}$ & 22 & 26,5 \\
\hline \multirow[t]{2}{*}{ Lateralidad } & Bilateral & 6 & 7,1 \\
\hline & Unilateral & 78 & 92,9 \\
\hline \multirow{2}{*}{$\begin{array}{l}\text { Antecedente de } \\
\text { malformación }\end{array}$} & Presencia de malformación & 6 & 7,1 \\
\hline & Sin malformación & 78 & 92,9 \\
\hline \multirow[t]{2}{*}{ Patología tumoral } & $\begin{array}{l}\text { Tumor de Wilms o nefroblastoma } \\
\text { con predominio blastemal }\end{array}$ & 23 & 53,5 \\
\hline & $\begin{array}{l}\text { Tumor de Wilms o nefroblastoma } \\
\text { sin predominio blastemal }\end{array}$ & 20 & 46,5 \\
\hline \multirow[t]{3}{*}{ Biopsia previa } & $\mathrm{Si}$ & 16 & 25,8 \\
\hline & No & 46 & 74,2 \\
\hline & Sin dato & 22 & \\
\hline \multirow{3}{*}{$\begin{array}{l}\text { Patología con presencia } \\
\text { de necrosis }\end{array}$} & Con necrosis & 11 & 17,7 \\
\hline & Sin necrosis & 51 & 82,3 \\
\hline & Sin dato & 22 & \\
\hline \multirow[t]{3}{*}{ Histología } & Histología desfavorable & 7 & 8,8 \\
\hline & Histología favorable & 73 & 91,2 \\
\hline & Sin dato & 4 & \\
\hline
\end{tabular}


Tabla 2. Tratamiento de los pacientes con tumor de Wilms de la población en estudio.

\begin{tabular}{|c|c|c|c|}
\hline Variable & Categoría & $\mathbf{n}$ & $\%$ \\
\hline \multirow[t]{2}{*}{ Resecabilidad quirúrgica } & Tumor irresecable & 33 & 39,3 \\
\hline & Tumor resecable & 51 & 60,7 \\
\hline \multirow[t]{3}{*}{ Lateralidad de la cirugía } & Unilateral & 78 & 96,3 \\
\hline & Bilateral & 3 & 3,7 \\
\hline & Sin dato & 3 & \\
\hline \multirow{2}{*}{$\begin{array}{l}\text { Momento de la nefrectomía } \\
\text { unilateral }\end{array}$} & Previa a quimioterapia & 51 & 65,4 \\
\hline & Posterior a quimioterapia & 27 & 34,6 \\
\hline \multirow[t]{3}{*}{ Contención tumoral } & Tumor no contenido & 35 & 43,2 \\
\hline & Tumor contenido & 46 & 56,8 \\
\hline & Sin dato & 3 & \\
\hline \multirow[t]{3}{*}{ Complicaciones quirúrgicas } & Cirugía con complicaciones & 10 & 12,3 \\
\hline & Cirugía sin complicaciones & 71 & 87,7 \\
\hline & Sin dato & 3 & \\
\hline \multirow[t]{3}{*}{ Protocolo de quimioterapia* } & NWTS & 58 & 70,7 \\
\hline & SIOP & 24 & 29,3 \\
\hline & Sin dato & 2 & \\
\hline \multirow{3}{*}{$\begin{array}{l}\text { Protocolo completo de } \\
\text { quimioterapia }\end{array}$} & No & 11 & 16,2 \\
\hline & $\mathrm{Si}$ & 57 & 83,8 \\
\hline & Sin dato & 16 & \\
\hline \multirow[t]{3}{*}{ Recibió radioterapia } & $\mathrm{Si}$ & 38 & 46,9 \\
\hline & No & 43 & 53,1 \\
\hline & Sin dato & 3 & \\
\hline \multirow[t]{3}{*}{ Presento recidiva } & $\mathrm{Si}$ & 20 & 27,4 \\
\hline & No & 53 & 72,6 \\
\hline & Sin dato & 11 & \\
\hline \multirow{3}{*}{$\begin{array}{l}\text { Presencia de trombo mural } \\
\text { en la tomografía }\end{array}$} & Con trombo tumoral en tomografía & 8 & 10,4 \\
\hline & Sin trombo tumoral en tomografía & 69 & 89,6 \\
\hline & Sin dato & 7 & \\
\hline \multirow{3}{*}{$\begin{array}{l}\text { Compromiso de ganglios } \\
\text { linfáticos en tomografía }\end{array}$} & Compromiso ganglios linfáticos & 23 & 31,1 \\
\hline & Sin Compromiso ganglios linfáticos & 51 & 68,9 \\
\hline & Sin dato & 10 & \\
\hline \multirow{3}{*}{$\begin{array}{l}\text { Presencia de metástasis a } \\
\text { distancia }\end{array}$} & Presencia de metástasis a distancia & 17 & 22,1 \\
\hline & Ausencia de metástasis a distancia & 60 & 77,9 \\
\hline & Sin dato & 7 & \\
\hline \multirow[t]{2}{*}{ Sobrevida a dos años } & Muerto & 18 & 29,5 \\
\hline & Vivo & 43 & 70,5 \\
\hline
\end{tabular}

* NWTS: National Wilms' Tumor Study; SIOP: Societe Internationale D’oncologie Pediatrique 
Tabla 3. Factores asociados a sobrevida a dos años en los pacientes con diagnóstico de tumor de Wilms.

\begin{tabular}{|c|c|c|c|c|c|c|c|c|}
\hline & \multirow{2}{*}{ Variables } & \multicolumn{2}{|c|}{ Muerto } & \multicolumn{2}{|c|}{ Vivo } & \multirow{2}{*}{ OR } & \multirow{2}{*}{$\mathrm{IC}_{95 \%}$} & \multirow{2}{*}{$\mathbf{p}$} \\
\hline & & $\mathbf{n}$ & $\%$ & $\mathbf{n}$ & $\%$ & & & \\
\hline \multirow{3}{*}{ Lugar de residencia } & Medellín y área metropolitana & 10 & 32,3 & 21 & 67,7 & 1 & & \\
\hline & Antioquia & 5 & 26,3 & 14 & 73,7 & 1,3 & $0,4-4,7$ & 0,657 \\
\hline & Otros departamentos & 2 & 20,0 & 8 & 80,0 & 1,9 & $0,3-10,7$ & 0,464 \\
\hline \multirow{2}{*}{ Sexo } & Masculino & 8 & 27,6 & 21 & 72,4 & 0,8 & $0,3-2,5$ & 0,754 \\
\hline & Femenino & 10 & 31,3 & 22 & 68,8 & 1 & & \\
\hline \multirow{2}{*}{ Presentación clínica } & $\begin{array}{l}\text { Masa abdominal y otra } \\
\text { sintomatología }\end{array}$ & 15 & 30,6 & 34 & 69,4 & 1,2 & $0,3-5,1$ & 0,827 \\
\hline & $\begin{array}{l}\text { Dolor abdominal u otra } \\
\text { sintomatología }\end{array}$ & 3 & 27,3 & 8 & 72,7 & 1 & & \\
\hline \multirow{2}{*}{ Lateralidad } & Bilateral & 3 & 60,0 & 2 & 40,0 & 4,1 & $0,6-27$ & 0,147 \\
\hline & Unilateral & 15 & 26,8 & 41 & 73,2 & 1 & & \\
\hline \multirow{2}{*}{$\begin{array}{l}\text { Antecedente de } \\
\text { malformación }\end{array}$} & Presencia de malformación & 1 & 20,0 & 4 & 80,0 & 0,6 & $0,06-5,5$ & 0,535 \\
\hline & Sin malformación & 17 & 30,4 & 39 & 69,6 & 1 & & \\
\hline \multirow{2}{*}{ Patología tumoral } & $\begin{array}{l}\text { Tumor de Wilms o nefroblastoma } \\
\text { con predominio blastemal }\end{array}$ & 5 & 33,3 & 10 & 66,7 & 3,2 & $0,5-20,4$ & 0,195 \\
\hline & $\begin{array}{l}\text { Tumor de Wilms o nefroblastoma } \\
\text { sin predominio blastemal }\end{array}$ & 2 & 13,3 & 13 & 86,7 & 1 & & \\
\hline \multirow{2}{*}{ Biopsia previa } & $\mathrm{Si}$ & 4 & 30,8 & 9 & 69,2 & 1,2 & $0,3-5$ & 0,518 \\
\hline & No & 9 & 26,5 & 25 & 73,5 & 1 & & \\
\hline \multirow{2}{*}{$\begin{array}{l}\text { Patología con presencia } \\
\text { de necrosis }\end{array}$} & Con necrosis & 1 & 14,3 & 6 & 85,7 & 0,4 & $0,05-4,1$ & 0,414 \\
\hline & Sin necrosis & 10 & 27,8 & 26 & 72,2 & & & \\
\hline \multirow{2}{*}{ Histología } & Histología desfavorable & 1 & 20,0 & 4 & 80,0 & 0,7 & $0,07-6,8$ & 0,614 \\
\hline & Histología favorable & 14 & 26,4 & 39 & 73,6 & & & \\
\hline \multirow{2}{*}{ Resecabilidad quirúrgica } & Tumor irresecable & 7 & 26,9 & 19 & 73,1 & 0,8 & $0,3-2,5$ & 0,703 \\
\hline & Tumor resecable & 11 & 31,4 & 24 & 68,6 & 1 & & \\
\hline \multirow{2}{*}{$\begin{array}{l}\text { Momento de la nefrectomía } \\
\text { unilateral }\end{array}$} & Previa a quimioterapia & 11 & 31,4 & 24 & 68,6 & 2,6 & $0,6-10,7$ & 0,178 \\
\hline & Posterior a quimioterapia & 3 & 15,0 & 17 & 85,0 & & & \\
\hline \multirow{2}{*}{ Cirugía bilateral } & Bilateral & 2 & 66,7 & 1 & 33,3 & 5,9 & $0,5-69,7$ & 0,181 \\
\hline & Unilateral & 14 & 25,5 & 41 & 74,5 & & & \\
\hline \multirow{2}{*}{ Contención tumoral } & Tumor no contenido & 7 & 31,8 & 15 & 68,2 & 1,4 & $0,4-4,5$ & 0,573 \\
\hline & Tumor contenido & 9 & 25,0 & 27 & 75,0 & & & \\
\hline \multirow{2}{*}{ Complicaciones quirúrgicas } & Cirugía con complicaciones & 4 & 50,0 & 4 & 50,0 & 3,2 & $0,7-14,6$ & 0,136 \\
\hline & Cirugía sin complicaciones & 12 & 24,0 & 38 & 76,0 & & & \\
\hline \multirow{2}{*}{ Protocolo de quimioterapia } & Protocolo SIOP & 4 & 21,1 & 15 & 78,9 & 0,5 & $0,1-2$ & 0,364 \\
\hline & Protocolo NWTS & 13 & 32,5 & 27 & 67,5 & & & \\
\hline \multirow{2}{*}{$\begin{array}{l}\text { Protocolo completo } \\
\text { de quimioterapia }\end{array}$} & No & 8 & 88,9 & 1 & 11,1 & 34 & $3,7-312,1$ & 0,000 \\
\hline & $\mathrm{Si}$ & 8 & 19,0 & 34 & 81,0 & & & \\
\hline Recibió radioterania & $\mathrm{Si}$ & 7 & 25,9 & 20 & 74,1 & 0,8 & $0,3-2,5$ & 0,708 \\
\hline ReciDio radioterapia & No & 10 & 30,3 & 23 & 69,7 & & & \\
\hline Procento recidive & $\mathrm{Si}$ & 11 & 78,6 & 3 & 21,4 & 35,7 & $6,9-184,2$ & 0,000 \\
\hline Presento reciarva & No & 4 & 9,3 & 39 & 90,7 & & & \\
\hline Presencia de trombo mural & Con trombo tumoral en tomografía & 3 & 42,9 & 4 & 57,1 & 2,1 & $0,4-10,5$ & 0,314 \\
\hline en la tomografía & Sin trombo tumoral en tomografía & 13 & 26,5 & 36 & 73,5 & & & \\
\hline Compromiso de ganglios & Con compromiso & 7 & 43,8 & 9 & 56,3 & 2,4 & $0,7-8,4$ & 0,139 \\
\hline linfáticos en la tomografía & Sin compromiso & 9 & 24,3 & 28 & 75,7 & & & \\
\hline Presencia de metástasis a & Presencia de metástasis & 6 & 46,2 & 7 & 53,8 & 2,5 & $0,7-9,1$ & 0,143 \\
\hline distancia & Ausencia de metástasis & 11 & 25,6 & 32 & 74,4 & & & \\
\hline
\end{tabular}


con tumores de riesgo intermedio (excluyendo los subtipos epitelial y estromal), la sobrevida libre de evento fue de $88-95 \%$ en los tumores menores de $500 \mathrm{ml}$ comparado con el 76-90 \% en los tumores mayores.

La histología es uno de los factores pronóstico más poderoso en tumor de Wilms. La anaplasia está presente en el 5-I0 \% y los estudios han mostrado que el predominio blastemal, como subtipo histológico, y la anaplasia, en especial si es difusa, son factores de peor pronóstico ${ }^{8,9}$.

En nuestra revisión, los factores que mostraron estar asociados a la sobrevida de manera estadísticamente significativa fueron la necesidad de cirugía por tumor bilateral, la recidiva y la quimioterapia incompleta. No se encontró que el tamaño tumoral, el estadio ni la histología estuvieran asociados con menor sobrevida. Cabe anotar que en nuestro medio no se realizan estudios moleculares de rutina, por lo que no puede analizarse en los resultados.

Los factores asociados a mortalidad tienen diversos resultados, que están ligados al nivel de desarrollo del país estudiado. Es así como, en estudios africanos se informa una incidencia de tumor de Wilms del $9 \%$ en la población pediátrica entre los o y 9 años, con una sobrevida del 52,7\%. Se han descrito factores como la desnutrición, la imposibilidad para acceder a una terapia óptima y la falta de seguros de salud como factores asociados a pobres resultados ${ }^{10-12}$. En otro estudio africano, Atanda y colaboradores encontraron que los mejores resultados estaban asociados con el estadio tumoral temprano al momento del diagnóstico, la histología no blastemal y recibir quimioterapia completa, este último factor fue similar al encontrado en nuestro estudio ${ }^{13}$. $\mathrm{Al}$ igual que en nuestros resultados, en África es más frecuente (hasta $72 \%$ ) que se presenten en estadios avanzados ${ }^{10,13-15}$.

Sangkhathat estudió pacientes en Tailandia, donde la incidencia es de $2,2 \%$, y encontró que los estadios de presentación más frecuentes eran el I y el III ( $38 \%$ ), con una sobrevida global del $65 \%$. Los factores asociados a los malos resultados fueron la falla en el tratamiento primario de pacientes en estadio I, el tamaño tumoral mayor de Io $\mathrm{cm}$ y la presentación con hematuria macroscópica. En nuestro estudio, el tamaño tumoral y la forma de presentación no tuvieron resultados con diferencias estadísticamente significativas; tampoco el género, la edad ni la histología ${ }^{16}$.

El estado de los nódulos reveló una asociación estadísticamente significativa con la sobrevida global. Sin embargo, podría pensarse que los nódulos positivos cambian el estadio de la enfermedad y esto puede representar el factor asociado más importante ${ }^{16}$. En otros estudios se ha encontrado que la densidad de los nódulos linfáticos es un predictor de supervivencia, lo que refuerza la importancia de tomar una adecuada muestra durante el acto quirúrgico, para mejorar la estadificación y alcanzar una posible reducción de la intensidad de la terapia en los casos de bajo riesgo ${ }^{17}$. Se ha mostrado que la falla en el muestreo ganglionar durante la cirugía no solo aumenta la probabilidad de recaída local, sino que es un indicador pronóstico independiente de menor supervivencia ${ }^{18 .}$

En países en vía de desarrollo, el retardo en el diagnóstico, la falta de recursos como quimioterapia e irradiación y la pobre adherencia terapéutica al tratamiento (lo cual puede estar asociado a toxicidad o a mal seguimiento) son factores cruciales en los pobres resultados de los niños con tumor de Wilms ${ }^{10,19}$; resultados similares a lo encontrado en nuestra revisión respecto a la quimioterapia incompleta.

En pacientes con tumor de Wilms estadio III con histología favorable, que normalmente tienen una buena sobrevida libre de enfermedad después del manejo con quimioterapia y radioterapia, se ha encontrado que los nódulos linfáticos y el estado de la $\mathrm{LOH}$, son factores altamente predictores de sobrevida libre de eventos, y que la invasión de los vasos intrarrenales no es un factor pronóstico independiente ${ }^{20,21}$.

En un estudio en Japón se encontró que los pacientes con histología blastemal tuvieron peor pronóstico comparado con los otros subtipos histológicos, por lo que sugieren que en estos casos 
las estrategias de tratamiento deben ser diferentes. Describieron que la sobrevida global a 5 años en el tipo epitelial fue del Ioo \%, en el estromal del $93 \%$ y en el mixto del $90 \%$, comparado con el tipo blastemal que fue del $65 \%$. Por otro lado, la sobrevida libre de eventos a 5 años fue del Ioo $\%$ en el tipo epitelial, $80 \%$ en el estromal, $87 \%$ en los mixtos y $52 \%$ en el blastemal ${ }^{22}$. En otro estudio japonés encontraron que el estadio inicial y el subtipo histológico fueron los únicos factores con diferencia estadísticamente significativa asociados a la recaída tumoral en niños con tumor de Wilms unilateral e histología favorable que fueron llevados a nefrectomía seguida de quimioterapia ${ }^{23}$.

En el protocolo UMBRELLA se validaron nuevos factores pronósticos, como el subtipo blastemal, el volumen del tumor y los marcadores moleculares, con el fin de determinar subgrupos de pacientes con peor pronóstico e incremento en los índices de recaídas ${ }^{24}$. También se han analizado factores quirúrgicos diferentes al muestreo ganglionar y a la ruptura intraoperatoria como factores que empeoran el pronóstico, y se ha descrito que dejar la fascia de Gerota no aumenta riesgo de recurrencia ${ }^{25}$.

La necesidad de intervención bilateral en nuestro estudio fue un factor que aumentó la probabilidad de morir, y si se suma una histología desfavorable, el impacto negativo sobre la supervivencia es mayor. Otros factores sumados a la bilateralidad como edad, sexo, estadio tumoral, la presencia de tumor meta o sincrónico, no han mostrado significancia estadística ${ }^{26}$.

Las limitaciones del presente estudio radican en que por ser un diseño transversal no se pueden establecer asociaciones de causalidad, aunque si establecer la relación de las variables con la sobrevida, y en que no se pudo seguir la totalidad de los pacientes.

\section{Conclusiones}

La sobrevida de nuestros niños con tumor de Wilms es menor que la reportada en la literatura mundial (70 \% versus más del $90 \%$ ), y los factores asociados a dicho desenlace fueron la falla en terminar el esquema de quimioterapia, la recidiva tumoral y la necesidad de cirugía bilateral.

\section{Consideraciones éticas}

Consentimiento informado: De acuerdo con la Resolución oo8430 de 1993 del Ministerio de Salud de Colombia, la investigación se clasifica como de bajo riesgo porque se basa en la revisión de datos de historias clínicas, por eso no se requiere el diligenciamiento del consentimiento informado. Previamente se solicitó autorización a los comités de investigaciones y de ética de ambas instituciones, las cuales avalaron la investigación.

Conflictos de interés: Los autores de la presente investigación no tiene ningún conflicto de intereses.

Financiación: esta es una investigación académica que no tiene patrocinio de ninguna empresa privada.

\section{Referencias}

I. Mejía F. Tumor de Wilms (nefroblastoma). En: Arango-Rave ME, Herrera-Toro N, Uribe-Restrepo FL, editores. Cirugía Pediátrica. Segunda edición. Medellín: Editorial Universidad de Antioquia, 20I7. p 365-37I.

2. Gurney JG, Severson RK, Davis S, Robison LL. Incidence of cancer in children in the UnitedStates. Sex-, race-, and I-year age-specific rates by histologic type. Cancer. I995;75:2I86-95. https://doi.org/IO.IOO2/IO97-OI 42(I99504I5)75:8<2I86::aid-cncr2820750825>3.0.co;2-f

3. Stiller CA, Parkin DM. International variations in the incidence of childhood renal tumours. Br J Cancer. I990;62:IO26-30. https://doi.org/IO.IO38/bjc.1990.432

4. Instituto Nacional de Cancerología - ESE. Anuario Estadístico 2015. Bogotá, 20I8. p. I20. Fecha de consulta: 20 de enero de 2020. Disponible en:

http://www.cancer.gov.co/files/libros/archivos/20I5

5. Herrera-Toro NH, Peña-Aguirre L, Arango-Rave ME. Tumor de Wilms: experiencia de 12 años en dos hospitales de alto nivel en Medellín, Colombia. Iatreia. 20I9;32:82-9I. https://doi.org/IO.I7533/udea.iatreia.I3

6. D’Angelo P, Di Cataldo A, Terenziani M, Bisogno G, Collini P, Di Martino M, et al. Factors possibly affecting prognosis in children with Wilms' tumor diagnosed before 24 months of age: A report from the Associazione Italiana Ematologia Oncologia Pediatrica (AIEOP) 
Wilms Tumor WorkingGroup. Pediatr Blood Cancer. 2017;64:I2-64. https://doi.org/IO.I002/pbc.26644

7. Aronson DC, Hadley GP. Age is not a prognostic factor in children with Wilms tumor beyond stage I in Africa. Pediatr Blood Cancer. 20I4;6I:987-9. https://doi.org/IO.IOO2/pbc.24948

8. Reinhard H, Semler O, Bürger D, Bode U, Flentje M, Göbel U, et al. Results of the SIOP 93-OI/GPOH trial and study for the treatment of patients with unilateral non metastatic Wilms tumor. Klin Padiatr. 2004;216:132-40. https://doi.org/IO.IO55/s-2004-822625

9. Dome JS, Perlman EJ, Graf N. Risk stratification for Wilms tumor: current approach and future directions. Am Soc Clin Oncol. 2014;34:215-23. https://doi.org/IO.I4694/EdBook_AM.20I4.34.215

Io. Uba AF, Chirdan LB. Childhood Wilms' tumour: prognostic factors in North Central Nigeria. West Afr J Med. 2007;26:222-5. https://doi.org/IO.43I4/wajm.v26i3.283I4

II. Ekenze SO, Agugua-Obianyo NEN, Odetunde OA. The challenge of nephroblastoma in a developing country. Ann Oncol. 2006;17:1598-I60o. https://doi.org/IO.Io93/annonc/mdli67

I2. Axt J, Abdallah F, Axt M, Githanga J, Hansen E, Lessan J, et al. Wilms tumor survival in Kenya. J Pediatr Surg. 2013;48:1254-62. https://doi.org/I0.IOI6/j.jpedsurg.2013.03.02I

I3. Atanda AT, Anyanwu L-JC, Atanda OJ, Mohammad AM, Abdullahi LB, Farinyaro AU. Wilms' tumour: Determinants of prognosis in an African setting. Afr J Paediatr Surg. 2015;12:17I-6. https://doi.org/I0.4IO3/0189-6725.170185

I4. Abubakar AM, Bwala JK, Abdur-Rahman LO, Chinda JY, Adeniran JO. Outcome of treatment of nephroblastoma in Nigerian children. Afr J Paediatr Surg. 2010;7:45-52.

I5. Osuoji RI, Williams OM, Ajai OT, Idika OC, Abolarinwa AA, Bankole MA. Wilms' tumour: Experience in a developing tertiary centre in Nigeria. East Cent Afr J Surg. 20II;I6:123-8.

I6. Sangkhathat S, Chotsampancharaen T, Kayasut K, Patrapinyokul S, Chiengkriwate P, Kitichet R, et al. Outcomes of pediatric nephroblastoma in southern Thailand. Asian Pac J Cancer Prev. 2008;9:643-7.

17. Saltzman AF, Carrasco A, Amini A, Aldrink JH, Dasgupta R, Gow KW, et al. Patterns of lymph node sampling and the impact of lymph node density in favorable histology Wilms tumor: An analysis of the national cancer database. J Pediatr Urol. 2018;I4:I6I. eI-I6I.e8. https://doi.org/I0.IoI6/j.jpurol.2017.09.025

I8. Zhuge Y, Cheung MC, Yang R, Koniaris LG, Neville HL, Sola JE. Improved survival with lymph node sampling in Wilms tumor. J Surg Res. 20II;167:eI99-203.

https://doi.org/IO.IOI6/j.jss.20I0.I2.026

I9. Kutluk T, Varan A, Büyükpamukçu N, Atahan L, Cağlar M, Akyüz C, et al. Improved survival of children with Wilms tumor. J Pediatr Hematol Oncol. 2006;28:423-6.

20. Breslow N, Sharples K, Beckwith JB, Takashima J, Kelalis PP, Green DM, et al. Prognostic factors in non metastatic, favorable histology Wilms' tumor. Results of the Third National Wilms' Tumor Study. Cancer. I991;68:2345-53. https://doi.org/IO.IOO2/IO97-OI42(I99II 20I)68:II<2345::aid-cncr282068IIO3>3.0.co;2-t

2I. Fernandez CV, Mullen EA, Chi Y-Y, Ehrlich PF, Perlman EJ, Kalapurakal JA, et al. Outcome and prognostic factors in stage III favorable-histology Wilms tumor: A report from the Children's Oncology Group Study ARENo532. J Clin Oncol. 20I8;36:254-6I. https://doi.org/IO.I200/JCO.2017.73.7999

22. Kinoshita Y, Suminoe A, Inada H, Yagi M, Yanai F, Zaizen Y, et al. The prognostic significance of blastemal predominant histology in initially resected Wilms' tumors: a report from the Study Group for Pediatric Solid Tumors in the Kyushu Area, Japan. J Pediatr Surg. 2012;47:2205-9.

https://doi.org/IO.IOI6/j.jpedsurg.2012.09.009

23. Aoba T, Urushihara N, Fukumoto K, Furuta S, Fukuzawa $\mathrm{H}$, Mitsunaga $\mathrm{M}$, et al. Relapse of unilateral favorable histology Wilms' tumor: significant clinicopathological factors. J Pediatr Surg. 2012;47:2210-15. https://doi.org/IO.IOI6/j.jpedsurg.20I2.09.0IO

24. Vujanić GM, Gessler M, Ooms AHAG, Collini P, Coulomb-l'Hermine A, D'Hooghe E, et al. The UMBRELLA SIOP-RTSG 2016 Wilms tumour pathology and molecular biology protocol. Nat Rev Urol. 20I8;15:693-70I. https://doi.org/IO.IO38/s4I585-0I8-0IOO-3

25. Zani A, Schiavetti A, Gambino M, Cozzi DA, Conforti A, Cozzi F. Long-term outcome of nephron sparing surgery and simple nephrectomy for unilateral localized Wilms tumor. J Urol. 2005;I73:946-8. https://doi.org/IO.IO97/oI.ju.0000I52580.9086I.d3

26. Sarhan OM, El-Baz M, Sarhan MM, Ghali AM, Ghoneim MA. Bilateral Wilms' tumors: single-center experience with 22 cases and literature review. Urology. 2010;76:946-5I.

https://doi.org/IO.IOI6/j.urology.2010.03.055 